\title{
1p and 19q Chromosomes Loss of Heterozygosity and Genetic Deletions Role in the Chemosensitivity of Oligodendrogliomas
}

\author{
Behzad Saberi* \\ Medical Research, Iran
}

*Corresponding author: Behzad Saberi, Medical Research, Esfahan, Iran.

\section{Opinion}

Oligodendroglioma as a glial tumor originating from oligodendrocytes, is identified by positive stain for myelin basic protein and characterized by 'fried-egg' appearance histologically. It would be a low-grade tumor with slow growing pattern, but anaplasia and aggressive behavior also can be occurred. Due to the hemorrhage in the tumor, sometimes the tumor will be symptomatic abruptly. CT and MRI scans can be used to determine the tumor as it presents as a hypointense T1-lesion on MRI scans and a nonenhanced hypodense lesion on CT scans. The presence of enhancement can be a sign of a mixed oligoastrocytoma or a more aggressive tumor. Sometimes calcifications can also be seen in the scans. Neuroimaging appearance, location and clinical presentation of the tumor, determine the management strategies. Since oligodendroglioma is a chemosensitive tumor, chemotherapy can be an effective treatment method for it. In case of possibility, surgical resection would be done generally for Oligodendroglioma. Genetic deletions or $1 \mathrm{p}$ and $19 \mathrm{q}$ chromosomes loss of heterozygosity can be seen in about sixty percent of oligodendrogliomas and this would be a chemosensitivity and favorable prognosis indicator. In case of the $1 \mathrm{p}$ and $19 \mathrm{q}$ chromosomes loss of heterozygosity, the overall survival and responsivity to chemotherapy would be more than ninety percent. PCV regimen and temozolomide are used in chemotherapy for oligodendrogliomas. In case of performing a limited biopsy or a subtotal resection, radiation therapy can have some advantages in the oligodendrogliomas survival, but in case of the possibility for total resection of the tumor, the role of the radiation therapy would be controversial [1-6].

\section{Acknowledgement}

None.

\section{Conflict of Interest}

No conflict of interest.

\section{References}

1. Cairncross JG, MacDonald DR, Ramsay DA (1992) Aggressive oligodendroglioma: a chemosensitive tumor. Neurosurgery 31: 78.

2. Cairncross G, Berkey B, Shaw E, Jenkins R, Scheithauer B, et al. (2006) Phase III trial of chemotherapy plus radiotherapy compared with radiotherapy alone for pure and mixed anaplastic oligodendroglioma: Intergroup Radiation Therapy Oncology Group Trial 9402. J Clin Oncol 24: $2707-2714$

3. Van den Bent MJ, Afra D, de Witte O, Ben Hassel M, Schraub S, et al. (2005) Long-term efficacy of early versus delayed radiotherapy for lowgrade astrocytoma and oligodendroglioma in adults: the EORTC 22845 randomized trial. Lancet 366: 985-990.

4. Hoang-Xuan K, Capelle L, Kujas M, Taillibert S, Duffau H, et al. (2004) Temozolomide as initial treatment for adults with low-grade oligodendrogliomas or oligoastrocytomas and correlation with chromosome 1p deletions. J Clin Oncol 22: 3133-3138.

5. Van den Bent MJ, Carpentier AF, Brandes AA, Sanson M, Taphoorn MJ, et al. (2006) Adjuvant procarbazine, lomustine, and vincristine improves progression-free survival but not overall survival in newly diagnosed anaplastic oligodendrogliomas and oligoastrocytomas: a randomized European Organisation for Research and Treatment of Cancer phase III trial. J Clin Oncol 24: 2715-2722.

6. Cairncross JG, Ueki K, Zlatescu MC, Lisle DK, Finkelstein DM, et al. (1998) Specific genetic predictors of chemotherapeutic response and survival in patients with anaplastic oligodendrogliomas. J Natl Cancer Inst 90: 1473-1479. 